\title{
RASTREAMENTO DE TRAJETÓRIAS POR SERVOVISÃO ADAPTATIVA
}

\author{
Antonio C. Leite* \\ toni@coep.ufrj.br \\ Fernando Lizarralde ${ }^{\dagger *}$ \\ fernando@coep.ufrj.br
}

\author{
Liu Hsu* \\ liu@coep.ufrj.br
}

Alessandro R. L. Zachi ${ }^{\ddagger}$

zachi@cefet-rj.br

*Programa de Engenharia Elétrica, COPPE-UFRJ

Cx. P. 68504, Rio de Janeiro, 21945-970 RJ, Brasil

†Departamento de Engenharia Eletrônica e de Computação, POLI

Universidade Federal do Rio de Janeiro

${ }^{\ddagger}$ Centro Federal de Educação Tecnológica Celso Suckow da Fonseca, CEFET/RJ

\begin{abstract}
In this work, a robotic visual servoing system using a fixed and uncalibrated camera is considered. Two adaptive controllers are proposed for visually tracking a moving target in the presence of camera parameters uncertainties. The first adaptive strategy uses the Hierarchy of Control method to solve the multivariable adaptive control problem. The second one is based on the symmetrization via factorization of the control matrix. The adaptive algorithms are robust in the sense that they present reduced sensitivity to kinematic uncertainties. Experimental results with a manipulator in a planar motion illustrate the robustness and viability of the proposed schemes.
\end{abstract}

KEYWORDS: Adaptive control, robotic manipulators, visual servoing.

\section{RESUMO}

Neste trabalho, considera-se um sistema robótico servovisual usando câmera fixa e não-calibrada. Dois controladores adaptativos são propostos para rastrear visualmente um alvo em movimento na presença de incertezas nos parâmetros da

\footnotetext{
Artigo submetido em 22/11/02

1a. Revisão em 20/02/03, 2a. Revisão em 18/06/03

Aceito sob recomendação do Ed. Assoc. Prof. Paulo E. Miyagi
}

câmera. A primeira estratégia adaptativa utiliza o método de Hierarquia de Controle para solucionar o problema de controle adaptativo multivariável. A segunda é baseada na simetrização via fatoração da matriz de controle. Os algoritmos adaptativos são robustos na medida em que apresentam reduzida sensibilidade à incertezas cinemáticas. Resultados experimentais com um manipulador em movimento planar ilustram a robustez e a viabilidade dos esquemas propostos.

PALAVRAS-CHAVE: Controle adaptativo, manipuladores robóticos, servovisão.

\section{INTRODUÇÃO}

O problema de rastreamento de alvos móveis nas coordenadas da imagem de uma câmera fixa e não-calibrada é um assunto de grande interesse sob o ponto de vista da teoria de controle, mesmo para o caso planar (Hutchinson et al., 1996).

Neste enfoque, o objetivo de controle é permitir que o efetuador de um manipulador robótico realize, através de servovisão, tarefas de rastreamento visual ao longo de trajetórias considerando incertos a rotação e os fatores de escala da câmera.

Em (Kelly, 1996) foi mostrado que um controlador PD (com parâmetros fixos) garante a regulação assintótica do sistema 
em presença de incerteza apenas na rotação da câmera. Sabese, inclusive, que o desempenho de controladores tipo PD pode ser melhorado adicionando-se um mecanismo de adaptação na lei de controle (Slotine e Li, 1991, Cap. 9).

Muitos esquemas adaptativos foram propostos para contornar a degradação de desempenho devido às incertezas de modelagem, particularmente com respeito à calibração da câmera (parâmetros intrínsecos) e aos parâmetros do robô (pârametros extrínsecos) (Koivo e Houshangi, 1991; Bishop e Spong, 1997; Hosoda et al., 1998). Entretanto, enquanto alguns trabalhos publicados consideram a interação dessas incertezas com algum algoritmo de calibração on-line justificado apenas de maneira ad hoc, outros requerem o conhecimento prévio dos parâmetros do sistema servovisual. Algumas exceções podem ser encontradas em publicações mais recentes, a saber, (Papanikolopoulos e Khosla, 1993; Hsu e Aquino, 1999; Kelly et al., 1999; Zergeroglu et al., 1999).

No presente trabalho serão apresentadas duas soluções para o rastreamento visual adaptativo direto de manipuladores robóticos planares usando câmera fixa e não-calibrada. Os algoritmos propostos são desenvolvidos para sistemas visuais look-and-move baseados em imagem (Hutchinson et al., 1996) e exigem apenas um reduzido conhecimento a priori dos parâmetros do sistema câmera/espaço de trabalho.

O primeiro esquema, denominado Controle Adaptativo Hierárquico, é baseado em um método proposto recentemente em (Hsu e Aquino, 1999; Hsu e Costa, 1999; Hsu e Lizarralde, 2000) que usa a abordagem da hierarquia de controle para solucionar o problema de controle adaptativo multivariável. O segundo esquema, denominado Controle Adaptativo Simétrico (Hsu e Lizarralde, 2000; Hsu et al., 2002), baseia-se em uma apropriada simetrização da matriz de controle, conduzindo a uma nova parametrização do controlador. Neste último esquema, a análise de estabilidade é bastante simples, porém uma sobre-parametrização é necessária. A eficácia dos métodos adaptativos é verificada através de simulações e ensaios experimentais em um robô manipulador real. Os resultados experimentais são obtidos através de uma Interface Gráfica com o Usuário desenvolvida em Tcl/Tk (Ousterhout, 1993).

\section{CONTROLE SERVOVISUAL}

Neste trabalho considera-se o problema de controle cinemático. Para este enfoque, a configuração do efetuador $x \in$ $\Re^{m}$ é dada pela transformação cinemática direta $x=F(q)$, sendo $q \in \Re^{n}$ o vetor de ângulos das juntas do manipulador. Assume-se aqui que o manipulador de interesse é planar e não-redundante, isto é, $m=n=2$. Generalizações para controle dinâmico poderiam ser feitas como em (Hsu e Aquino, 1999).
A velocidade do efetuador em termos das velocidades das juntas é obtida através da relação:

$$
\dot{x}=J(q) \dot{q}
$$

onde $J(q)=\frac{\partial F(q)}{\partial q} \in \Re^{2 \times 2}$ é o Jacobiano do manipulador. Então, considerando $\dot{q}_{i}$ como sendo a entrada de controle $u_{i}(i=1, \cdots, n)$, obtém-se o seguinte sistema

$$
\dot{x}=J(q) u \text {. }
$$

A lei de controle cartesiana $v$ pode ser transformada em sinal de controle para as juntas através da seguinte relação:

$$
u=J^{-1}(q) v
$$

Utilizando uma câmera CCD monocular, fixa e nãocalibrada, com eixo ótico perpendicular (Vide (Hsu et al., 2001) e suas referências para o caso não-perpendicular) ao espaco de trabalho do robô, a transformação câmera/espaço de trabalho pode ser representada por (Hutchinson et al., 1996):

$$
x_{c}=K_{p} x+x_{c 0},
$$

com

$$
K_{p}=\frac{f}{f-Z}\left[\begin{array}{cc}
\alpha_{1} & 0 \\
0 & \alpha_{2}
\end{array}\right]\left[\begin{array}{rr}
\cos (\phi) & -\sin (\phi) \\
\sin (\phi) & \cos (\phi)
\end{array}\right] \text {, }
$$

onde $x_{c}$ é a posição do efetuador no sistema de coordenadas da câmera; $x_{c 0}$ é um termo constante que representa a posição do sistema de coordenadas da câmera em relação ao sistema de coordenadas do robô; $K_{p}$ é a matriz de transformação do espaço operacional para o espaço da câmera; $\phi$ é o ângulo de rotação da câmera em torno do eixo ótico; $f$ é a distância focal da câmera; $Z<0$ é a profundidade relativa medida entre o plano da imagem e o espaço de trabalho do robô (em geral $Z \gg f$ ) e $\alpha_{1}, \alpha_{2}>0$ são fatores de escala da câmera (pixels/mm). Sem perda de generalidade, considerase que $x_{c 0}=0$ (Hsu e Aquino, 1999). Portanto, o problema do controle cartesiano no sistema de coordenadas da câmera é descrito por:

$$
\dot{x}_{c}=K_{p} v,
$$

onde $v^{T}=\left[\begin{array}{ll}v_{1} & v_{2}\end{array}\right]$ e $K_{p}$ é incerto.

\section{MRAC SERVOVISUAL}

Na abordagem de controle adaptativo por modelo de referência (MRAC) direto, o modelo pode ser especificado por

$$
\dot{x}_{c d}=-\Lambda x_{c d}+\Lambda r_{c},
$$

onde $r_{c} \in \Re^{2}$ é o sinal de referência, assumido uniformemente limitado $\forall t$, e $x_{c d} \in \Re^{2}$ é a trajetória desejada para o efetuador no plano da câmera. Por simplicidade, assume-se que $\Lambda=\lambda I$. 
É possível modificar a estratégia adotada em (Hsu e Aquino, 1999) para introduzir o erro da imagem diretamente na lei de controle, mesmo no caso em que a adaptação esteja desligada. Deste modo, de acordo com (5), a lei de controle ideal $v^{*}$ que promove o casamento com o modelo (6), é dada por:

$$
v=v^{*}=\lambda K_{p}^{-1}\left(r_{c}-x_{c}\right) .
$$

Substituindo $r_{c}$, obtido a partir de (6), em (7) vem:

$$
v^{*}=K_{p}^{-1}\left[\dot{x}_{c d}+\Lambda\left(x_{c d}-x_{c}\right)\right] .
$$

Então, de acordo com (2), tem-se que

$$
u=\left(K_{p} J(q)\right)^{-1}\left[\dot{x}_{c d}+\Lambda\left(x_{c d}-x_{c}\right)\right],
$$

onde $J_{v}=K_{p} J(q)$ é comumente denominado de Jacobiano da imagem (Hutchinson et al., 1996).

A partir de (5) e (6), definindo o erro da imagem $e_{c}=x_{c}-$ $x_{c d}$, obtém-se o seguinte sistema de erro

$$
\dot{e}_{c}=-\lambda e_{c}+K_{p} v-\lambda\left(r_{c}-x_{c}\right),
$$

ou, ainda,

$$
\dot{e}_{c}=-\lambda e_{c}+K_{p} \tilde{v},
$$

onde $\tilde{v}=v-v^{*}$, e $K_{p}=\left[k_{i j}\right]$ para $i, j=1,2$.

\subsection{Adaptação via Hierarquia de Controle}

Da expressão de $v^{*}$ (vide (7)) verifica-se que a parametrização linear usual para a lei de controle é dada por

$$
v=P\left(r_{c}-x_{c}\right),
$$

com $P \in \Re^{2 \times 2}$ sendo a matriz de parâmetros.

Para o caso de plantas SISO sabe-se que para solucionar o problema de controle adaptativo o sinal do ganho de alta frequência deve ser, em geral, conhecido. A maior dificuldade no caso MIMO é a generalização da condição de conhecimento do sinal, tendo em vista a ocorrência de ganhos matriciais. Os resultados em (Narendra e Annaswamy, 1989; Ioannou e Sun, 1996) requerem condições restritivas sobre $K_{p}$ difíceis de serem atendidas na prática. Em particular, o método apresentado em (Ioannou e Sun, 1996) requer a existência de uma matriz $S \in \Re^{2 \times 2}$ tal que $K_{p} S=\left(K_{p} S\right)^{T}>0$, que segundo os mesmos autores é equivalente ao conhecimento do sinal de $K_{p}$ no caso SISO. No entanto, esta condição de existência é muito restritiva por tratar-se de uma restrição de igualdade. Além disso, tal restrição é não-genérica uma vez que pode ser violada para qualquer incerteza em $K_{p}$. No caso do problema de servovisão verifica-se que não existe uma matriz $S$ satisfazendo esta restrição, e consequentemente, o método proposto em (Ioannou e Sun, 1996) não pode ser aplicado.
Por outro lado, aplicando o método de Hierarquia de Controle (HC) introduzido recentemente em (Hsu e Aquino, 1999; Hsu e Costa, 1999; Hsu e Lizarralde, 2000), é possível obter uma parametrização linear conveniente para o vetor de controle $v^{*}$ em (7). É válido ressaltar que as condições requeridas para a utilização desta estratégia são menos restritivas do que as exigidas em (Narendra e Annaswamy, 1989; Ioannou e Sun, 1996).

O método de Hierarquia de Controle consiste em projetar a lei de controle considerando alguma hierarquia préviamente escolhida. Expandindo (8) elemento a elemento tem-se que:

$$
\begin{aligned}
& \dot{e}_{c 1}=-\lambda e_{c 1}+k_{11} v_{1}+k_{12} v_{2}-\lambda\left(r_{c 1}-x_{c 1}\right), \\
& \dot{e}_{c 2}=-\lambda e_{c 2}+k_{21} v_{1}+k_{22} v_{2}-\lambda\left(r_{c 2}-x_{c 2}\right) .
\end{aligned}
$$

Assumindo que a hierarquia escolhida ${ }^{1}$ é $e_{c 2} \rightarrow e_{c 1}$, inicialmente projeta-se $v_{2}$ de forma que $e_{c 2}=0$ seja alcançado assintoticamente; em seguida, projeta-se $v_{1}$ para que $e_{c 1}=0$ também seja alcançado assintoticamente ou, pelo menos, no sentido de média quadrática. Fazendo $e_{c 2} \equiv 0$ em (11), o sinal de controle $v_{2}$ resultante é dado por:

$$
v_{2}=v_{2}^{*}=\lambda k_{22}^{-1}\left(r_{c 2}-x_{c 2}\right)-k_{22}^{-1} k_{21} v_{1},
$$

conferindo à $e_{c 1}$ a seguinte dinâmica:

$$
\begin{aligned}
\dot{e}_{c 1}= & -\lambda e_{c 1}+k_{22}^{-1} \operatorname{det}\left(K_{p}\right) v_{1} \\
& +\lambda k_{12} k_{22}^{-1}\left(r_{c 2}-x_{c 2}\right)-\lambda\left(r_{c 1}-x_{c 1}\right) .
\end{aligned}
$$

Agora, fazendo $e_{c 1} \equiv 0$ em (13), obtém-se a seguinte lei para $v_{1}$

$$
\begin{aligned}
v_{1}^{*}= & \lambda \operatorname{det}\left(K_{p}^{-1}\right) k_{22}\left(r_{c 1}-x_{c 1}\right) \\
& -\lambda \operatorname{det}\left(K_{p}^{-1}\right) k_{12}\left(r_{c 2}-x_{c 2}\right) .
\end{aligned}
$$

Com base nas equações (12) e (14), a seguinte parametrização é natural:

$$
\begin{aligned}
& v_{1}=\theta_{11}\left(r_{c 1}-x_{c 1}\right)+\theta_{12}\left(r_{c 2}-x_{c 2}\right), \\
& v_{2}=\theta_{22}\left(r_{c 2}-x_{c 2}\right)+\theta_{23} v_{1} .
\end{aligned}
$$

Os parâmetros ideais associados a parametrização dada por (15) são computados a partir de (12) e (14):

$$
\begin{array}{rlrl}
\theta_{11}^{*} & =\lambda \operatorname{det}\left(K_{p}^{-1}\right) k_{22}, \theta_{12}^{*} & =-\lambda \operatorname{det}\left(K_{p}^{-1}\right) k_{12}, \\
\theta_{22}^{*} & =\lambda k_{22}^{-1}, & \theta_{23}^{*} & =-k_{22}^{-1} k_{21} .
\end{array}
$$

Agora, definem-se os vetores de parâmetros $\theta_{1}=\left[\begin{array}{ll}\theta_{11} & \theta_{12}\end{array}\right]^{T}$ e $\theta_{2}=\left[\begin{array}{ll}\theta_{21} & \theta_{22}\end{array}\right]^{T}$, e os vetores regressores

$$
w_{1}=\left[\begin{array}{c}
r_{c 1}-x_{c 1} \\
r_{c 2}-x_{c 2}
\end{array}\right], \quad w_{2}=\left[\begin{array}{c}
r_{c 2}-x_{c 2} \\
\theta_{1}^{T} \omega_{1}
\end{array}\right],
$$

${ }^{1}$ A hierarquia deve ser escolhida de forma que os menores principais $\Delta_{i}(i=1,2)$, de $K_{p}$ sejam diferentes de zero e tenham sinais conhecidos (Hsu e Costa, 1999). 
ou ainda,

$$
\begin{aligned}
w_{1} & =w_{m 1}-e_{c}, \quad w_{m 1}=r_{c}-x_{c d}, \\
w_{2} & =w_{m 2}-\left[\begin{array}{cc}
e_{c 2} \\
\theta_{1}^{T} e_{c}+\tilde{\theta}_{1}^{T} w_{m 1}
\end{array}\right], \\
w_{m 2} & =\left[\begin{array}{c}
r_{c 2}-x_{c d 2} \\
\theta_{1}^{* T} w_{m 1}
\end{array}\right],
\end{aligned}
$$

onde $w_{m 1}$ e $w_{m 2}$ são vetores uniformemente limitados, introduzidos apenas para auxiliar a análise de estabilidade, e $\tilde{\theta}_{1}=\theta_{1}-\theta_{1}^{*}$. Portanto, a partir das equações (15), (17), (18) e (19), pode-se escrever $v_{1}=\theta_{1}^{T} w_{1}$ e $v_{2}=\theta_{2}^{T} w_{2}$, e obter as equações de erro em uma forma mais compacta:

$$
\begin{aligned}
& \dot{e}_{c 1}=-\lambda e_{c 1}+k_{p 1} \tilde{\theta}_{1}^{T} w_{1}+k_{12} \tilde{\theta}_{2}^{T} w_{2}, \\
& \dot{e}_{c 2}=-\lambda e_{c 2}+k_{p 2} \tilde{\theta}_{2}^{T} w_{2},
\end{aligned}
$$

onde $\tilde{\theta}_{i}=\theta_{i}-\theta_{i}^{*} \quad(i=1,2)$ são os vetores de erro paramétrico, $k_{p 1}=k_{22}^{-1} \operatorname{det}\left(K_{p}\right)$ e $k_{p 2}=k_{22}$. Para o projeto das leis de adaptação, convém notar que a equação de $\dot{e}_{c 2}$ (21) está na forma padrão para a definição de uma lei tipo gradiente pura para a adaptação de $\theta_{2}$. Por outro lado, na equação de $\dot{e}_{c 1}(20)$ há um termo adicional $k_{12} \tilde{\theta}_{2}^{T} w_{2}$ no formato de perturbação de entrada. A fim de evitar a ocorrência de drift nos valores de $\theta_{1}$ e garantir robustez à perturbações, adotase uma lei gradiente modificada por um operador projeção (Ioannou e Sun, 1996), isto é:

$$
\begin{aligned}
& \dot{\theta}_{1}=\dot{\tilde{\theta}}_{1}=\mathcal{P}\left\{-\operatorname{sign}\left(k_{p 1}\right) \gamma w_{1} e_{c 1}\right\}, \\
& \dot{\theta}_{2}=\dot{\tilde{\theta}}_{2}=-\operatorname{sign}\left(k_{p 2}\right) \gamma w_{2} e_{c 2},
\end{aligned}
$$

onde $\gamma>0$, e o operador de projeção $\mathcal{P}$ é usado para garantir que o parâmetro $\theta_{1}$ continue uniformemente limitado dentro de algum conjunto compacto convexo contendo o vetor de parâmetros $\theta_{1}^{*}$. Por simplicidade, será assumido de agora em diante que esse conjunto é uma esfera fechada com centro na origem do espaço de parâmetros ${ }^{2}$. Note que o operador $\mathcal{P}$ garante a limitação uniforme de $\theta_{1}$.

\subsection{Análise de Estabilidade}

A prova de estabilidade do esquema adaptativo hierárquico segue a referência (Hsu e Aquino, 1999). Antes de prosseguir, é necessário definir a seguinte classe de funções:

Definição 1 (Ioannou e Sun, 1996) Seja $\eta:[0, \infty) \rightarrow R^{n}, \psi:$ $[0, \infty) \rightarrow R^{+}$, onde $\eta \in \mathcal{L}_{2 e}, \psi \in \mathcal{L}_{1 e}$ e considere a classe de funções

$\mathcal{S}(\psi)=\left\{\eta, \psi: \int_{t}^{t+T}\|\eta(\tau)\|_{2}^{2} d \tau \leq c_{0} \int_{t}^{t+T} \psi(\tau) d \tau+c_{1}, \forall t\right\}$

\footnotetext{
${ }^{2}$ É mais conveniente escolher algum vetor de parâmetro nominal para definir o centro da esfera. Entretanto, isto poderia levar a cálculos mais complexos.
}

finitas. Diz-se que $\eta$ é $\psi$-pequeno no sentido de média quadrática se $\eta \in \mathcal{S}(\psi)$.

Os seguintes lemas estão relacionados aos problemas de estabilidade de controle adaptativo de plantas SISO com grau relativo 1 com possíveis perturbações de saída, $d(t)$, respectivamente. Considere o sistema adaptativo com lei gradiente

$$
\begin{aligned}
& \dot{e}=-\lambda e+k_{p} \phi_{s}^{T} w_{s}(t), \\
& \dot{\phi}=\mathcal{P}_{e}\left[-\operatorname{sign}\left(k_{p}\right) \gamma w_{s}(t)(e+d(t))\right],
\end{aligned}
$$

onde $e \in \Re ; \phi_{s} \in \Re^{m} ; \lambda, \gamma>0 ; w_{s}(t) \in \mathcal{L}_{\infty}$ é contínuo por parte em $t \mathrm{e} \mathcal{P}_{e}$ denota o operador de projeção estendido, ou seja, aquele que engloba o operador identidade (sem projeção).

Lema 1 Considere o sistema (24)-(25) sem perturbação, isto é, $d \equiv 0$ e sem projeção. Então, as seguintes propriedades de estabilidade são válidas: (1) $\lim _{t \rightarrow \infty} e(t)=0$, $\phi_{s}(t) \in \mathcal{L}_{\infty}, e(t) \in L_{2} \cap \mathcal{L}_{\infty} ;(2)$ se $w_{s}(t), \dot{w}_{s}(t) \in \mathcal{L}_{\infty} e$ $w_{s}(t)$ é persistentemente excitado $(P E)$, então a origem do espaço de erros $\left(e=0, \phi_{s}=0\right)$ é globalmente exponencialmente estável.

Prova: A propriedade 1 é mostrada através da Função de Lyapunov $2 V\left(e, \phi_{s}\right)=e^{2}+\left|k_{p}\right| \frac{\phi_{s}^{T} \phi_{s}}{\gamma}$, que em virtude de (24), tem derivada no tempo $\dot{V}=-\lambda e^{2}$. Para mais detalhes da prova, veja (Ioannou e Sun, 1996, Sec. 6.4.1). A propriedade 2 é um resultado padrão (veja (Sastry e Bodson, 1989, Teorema 2.6.5)).

Lema 2 Considere o sistema (24)-(25), com perturbação de saída $d(t)$ uniformemente limitada e operador de projeção $\mathcal{P}$ com relação a alguma esfera finita contendo $\phi_{s}=0$. Então, verifica-se as seguintes propriedades de estabilidade: (1) $\phi_{s}(t) \in \mathcal{L}_{\infty}, e(t) \in \mathcal{S}(|d|) \cap \mathcal{L}_{\infty}$; (2) se $w_{s}(t), \dot{w}_{s}(t) \in$ $\mathcal{L}_{\infty}$ e $w_{s}(t)$ é persistentemente excitado (PE), então, para $\varepsilon=\left[e, \phi_{s}^{T} / \sqrt{\gamma}\right]^{T}$, tem-se o seguinte limitante:

$$
\|\varepsilon(t)\| \leq K_{0} e^{-\zeta t}\|\varepsilon(0)\|+K_{1} e^{-\zeta t} *|d(t)|,
$$

onde “*” denota o operador de convolução. Se, além disso, $d \in \mathcal{L}_{2}$ então todos os componentes de $\varepsilon$ são também $\mathcal{L}_{2} e$ tendem a zero quando $t \rightarrow \infty$ (prova: vide (Hsu e Aquino, 1999)).

Considere o sistema adaptativo consistindo de (5), com o controlador de rastreamento visual adaptativo definido por (6), (15), (22) e (23). Assume-se que o sinal de referência $r_{c}(t)$ é contínuo por partes e uniformemente limitado em norma e que o operador de projeção em $\dot{\theta}_{1}$ é definido com relação a uma esfera fechada $\mathcal{B}=\left\{\theta_{1}: \theta_{1} \in \Re^{m},\left\|\theta_{1}\right\| \leq M\right\}$ com $M>\left\|\theta_{1}^{*}\right\|$. Por conveniência, definindo-se uma nova 
variável $z_{1}$ tal que $e_{c 1}=z_{1}+\alpha e_{c 2}$, onde $\alpha=\frac{k_{12}}{k_{22}}$, a partir de (20) e (21) pode-se escrever

$$
\dot{z}_{1}=-\lambda z_{1}+k_{p 1} \tilde{\theta}_{1}^{T} w_{1}
$$

Então, o seguinte teorema pode ser enunciado:

Teorema 1 Se o ângulo de rotação $\phi \in(-\pi / 2, \pi / 2)$ e $\theta_{1}(0) \in \mathcal{B}:$ (1) todos os sinais do sistema são uniformemente limitados e $\lim _{t \rightarrow \infty} e_{c 2}(t)=0, e_{c 2}(t) \in \mathcal{L}_{2} \cap \mathcal{L}_{\infty}$, $e_{c 1}(t)=z_{1}(t)+\alpha e_{c 2}(t), z_{1}(t) \in \mathcal{S}\left(\left|e_{c 2}\right|\right) \cap \mathcal{L}_{\infty}$, para alguma constante $\alpha$; (2) se as seguintes hipóteses forem consideradas: (a) $r_{c}, \dot{r}_{c} \in \mathcal{L}_{\infty}$; (b) $w_{m 1}=r_{c}-x_{c d}$ é persistentemente excitado (PE); e (c) $w_{m 2}=\left[\left(r_{c 2}-x_{c d 2}\right)\left(\theta_{1}^{* T} w_{m 1}\right)\right]$ é persistentemente excitado (PE); então o sistema de erros completo, com estado $\left[e_{c 1}, e_{c 2}, \tilde{\theta}_{1}^{T}, \tilde{\theta}_{2}^{T}\right]^{T}$, é exponencialmente estável em qualquer esfera fechada (finita) ${ }^{3}$.

Prova: Nota-se primeiro que $\operatorname{det}\left(K_{p}\right)>0$ e, para $|\phi|<$ $\frac{\pi}{2}, k_{p 1}, k_{p 2}>0$. Desde que os vetores regressores $w_{1}, w_{2}$ são não-limitados a priori, e como $w_{1}, w_{2}$ dependem do erro $e_{c}$, deve-se mostrar que os mesmos são u.l. para aplicar os Lemas 1 e 2, formulados na seção 3.2 .

O sistema (21)(23) é localmente Lipschitz-continuo em toda parte no espaço de estado do erro. Deste modo, a partir de qualquer condição inicial no tempo $t_{0}$, existe $t_{f}>0$ tal que a solução do sistema existe em $\mathcal{I}=\left(t_{0}, t_{0}+t_{f}\right)$, e se a solução não pode ser continuada para $t \geq t_{0}+t_{f}$, $\mathcal{I}$ é dito ser seu máximo intervalo de existência. Então, é conhecido (Michel e Miller, 1982, Corolário 3.2) que se $t_{f}=\infty$ ou não, então o estado deve tornar-se ilimitado quando $t \rightarrow t_{0}+t_{f}$. É possível mostrar que para o sistema considerado $t_{f}=\infty$, isto é, todos os sinais do sistema são definidos para todo $t \mathrm{e}$ também são uniformemente limitados.

Primeiramente, considere o subsistema (21)(23). A função de Lyapunov

$$
2 V_{2}=e_{c 2}^{2}+\gamma^{-1} k_{p 2} \tilde{\theta}_{2}^{T} \tilde{\theta}_{2}
$$

tem derivada $\dot{V}_{2}=-\lambda e_{c 2}^{2} \leq 0$ em relação a este subsistema. Segue que $\varepsilon_{2}$ é uniformemente limitado (u.l.) por uma constante independente de $t_{0}, t_{f}, w_{2}, \forall t \in \mathcal{I}$. Por outro lado, considerando o subsistema (22)(26), a função de Lyapunov

$$
2 V_{1}=x_{1}^{2}+\gamma^{-1} k_{p 1} \tilde{\theta}_{1}^{T} \tilde{\theta}_{1}
$$

tem derivada limitada por (Hsu e Aquino, 1999)

$$
\dot{V}_{1} \leq-\lambda x_{1}^{2}+\alpha\left|k_{p 1}\right| \tilde{\theta}_{1}^{T} w_{1} e_{c 2}
$$

Contudo, $w_{1}$ é dado por (17), onde $w_{m 1}$ é u.l.. Uma vez que já foi mostrado que $e_{c 2}$ é u.l. e $\tilde{\theta}_{1}$ também é u.l. devido a $\mathcal{P}$,

\footnotetext{
${ }^{3}$ Vide (Sastry e Bodson, 1989, Teorema 3.8.1) para detalhes sobre a diferença entre este conceito e estabilidade exponencial global.
}

pode-se completar os quadrados e obter um novo limitante

$$
\dot{V}_{1} \leq-\frac{3}{4} \lambda x_{1}^{2}+k_{1}^{2}
$$

onde $k_{1}$ é uma constante dependente dos limites de $e_{c 2}, \tilde{\theta}_{1} \mathrm{e}$ $w_{m 1}$, sendo válido $\forall t \in \mathcal{I}$. Desde que $\tilde{\theta}_{1}$ é u.l., da expressão $V_{1}$ um limite adicional é obtido

$$
\dot{V}_{1} \leq-\frac{3}{2} V_{1}+k_{2}^{2}
$$

onde $k_{2}$ é uma constante, $\forall t \in \mathcal{I}$. Invocando o Lema de Comparação (Khalil, 1996, Lema 2.5), segue imediatamente que $V_{1}$ e portanto $z_{1}, e_{c 1}, \tilde{\theta}_{1}$ são todos u.l. em $\mathcal{I}$. Portanto, $t_{f}=\infty$ e todos os sinais do sistema são u.l. para $\forall t$. Então, aplicando os Lemas 1 e 2 ao subsistema (22)(26) e (21)(23) respectivamente, a propriedade 1 está provada.

Com o intuito de provar a propriedade 2, primeiramente deve-se invocar a propriedade 2 do Lema 2 baseada na conclusão que $d=\alpha e_{c 2} \in \mathcal{L}_{2}$ e sobre as hipóteses (a) e (b) de limitação e PE de $w_{m 1}$. De (17), as hipóteses anteriores implicam que $w_{1}$ e $\dot{w}_{1}$ também são u.l.. Além disso, da propriedade $1, e_{c 2} \in \mathcal{L}_{2}$ e $e_{c 1} \in S\left(\left|e_{c 2}\right|\right)$. Por isso, se $w_{m 1}$ é PE então $w_{1}$ também é PE (ver (Hsu e Costa, 1999)). Do Lema 2 , conclui-se que $\tilde{\theta}_{1} \in \mathcal{L}_{2}$, que implica $\tilde{\theta}_{1}^{T} w_{m 1} \in \mathcal{L}_{2}$. Assim, de (18) pode-se concluir similarmente que se $w_{m 2}$ é PE então $w_{2}$ também é PE. Portanto, o subsistema (21)(23) é exponencialmente estável, isto é, existe $c_{2}>0, \lambda_{2}>0$ tal que o estado $\varepsilon_{2}:=\left[\begin{array}{ll}e_{c 2} & \tilde{\theta}_{2}^{T}\end{array}\right]^{T}$ é limitado por

$$
\left\|\varepsilon_{2}(t)\right\| \leq c_{2} e^{-\lambda_{2} t}\left\|\varepsilon_{2}(0)\right\|
$$

Por outro lado, notando que $|d(t)| \leq|\alpha|\left\|\varepsilon_{2}(t)\right\|$, tem-se a partir da propriedade 2 do Lema 2 aplicado para (22)(26),

$$
\begin{aligned}
\left\|\varepsilon_{1}(t)\right\| & \leq c_{0} e^{-\lambda_{2} t}\left\|\varepsilon_{1}(0)\right\|+c_{1}\left(e^{-\lambda_{1} t} * e^{-\lambda_{2} t}\right)\left\|\varepsilon_{2}(0)\right\| \\
& \leq c_{3} e^{-\lambda_{3} t}\|\varepsilon(0)\|
\end{aligned}
$$

onde, $c_{0}, c_{1}, c_{3}, \lambda_{2}$ e $\lambda_{3}$ são constantes positivas, $\varepsilon_{1}:=$ $\left[\begin{array}{ll}x_{1} & \tilde{\theta}_{1}^{T}\end{array}\right]^{T}$ como definido antes do Teorema 1 , e $\varepsilon^{T}:=$ $\left[\begin{array}{ll}\varepsilon_{1}^{T} & \varepsilon_{2}^{T}\end{array}\right]$ sendo o estado completo do sistema (22)(26) e (21)(23). Agora combinando as inequações (32) e (33) obtém-se a propriedade que caracteriza a estabilidade exponencial, a saber, $\|\varepsilon(t)\| \leq c e^{-\lambda_{3} t}\|\varepsilon(0)\|$. Isto prova a propriedade 2 do Teorema.

É válido notar que a condição PE no Teorema 1 é dada diretamente em função do vetor de referência $r_{c}(t)$ e da trajetória de referência $x_{c d}(t)$. De fato, a condição PE foi necessária para $w_{m 1}$ e $w_{m 2}$ como definido em (17)(18). Nota-se também que o nível de PE pode ser arbitrariamente pequeno.

Fato 1 Embora o Teorema 1 forneça um resultado de estabilidade global, isto pode não ser verdadeiro quando considerase a lei de controle dada por (2). O resultado de estabilidade é mantido desde que a configuração do robô permaneça o tempo todo longe das singularidades do Jacobiano. 


\section{ADAPTAÇÃO VIA SIMETRIZAÇÃO}

Um método mais simples, similar ao esquema de controle robusto proposto em (Zergeroglu et al., 1999), consiste na Simetrização de $K_{p}$ (SK) juntamente com uma apropriada redefinição da parametrização do vetor de controle. Este método baseia-se no fato de que é possível determinar uma matriz triangular superior $T$ tal que $\left(K_{p} T\right)=\left(K_{p} T\right)^{T}=S>0$, desde que $k_{11} \neq 0$ e $\operatorname{det}\left(K_{p}\right) \neq 0$ (Hsu et al., 1999). Uma vez que $T$ esteja definida, é possível reescrever (8) na forma:

$$
\dot{e}_{c}=-\lambda e_{c}+S\left[T^{-1} v-\lambda S^{-1}\left(r_{c}-x_{c}\right)\right] .
$$

Se $k_{11}>0$ e $\operatorname{det}\left(K_{p}\right)>0$, então $T$ (e $T^{-1}$ ) pode ser escolhido com elementos diagonais unitários, ou seja,

$$
T=\left[\begin{array}{cc}
1 & t_{12} \\
0 & 1
\end{array}\right], \quad T^{-1}=\left[\begin{array}{cc}
1 & -t_{12} \\
0 & 1
\end{array}\right] .
$$

Escrevendo $S$ em função dos elementos de $K_{p}$,

$$
S=\left[\begin{array}{ll}
k_{11} & k_{11} t_{12}+k_{12} \\
k_{21} & k_{21} t_{12}+k_{22}
\end{array}\right],
$$

e avaliando a condição de simetria $k_{21}=k_{11} t_{12}+k_{12}$, chegase à

$$
t_{12}=\frac{k_{21}-k_{12}}{k_{11}} .
$$

Substituindo (37) em (36), pode-se reescrever $S$ como

$$
S=\left[\begin{array}{ll}
k_{11} & k_{21} \\
k_{21} & s_{22}
\end{array}\right], \quad s_{22}=\frac{k_{21}^{2}+\operatorname{det}\left(K_{p}\right)}{k_{11}} .
$$

Da mesma forma, sabendo que $\operatorname{det}(S)=\operatorname{det}\left(K_{p}\right)$, tem-se que

$$
S^{-1}=\operatorname{det}\left(K_{p}^{-1}\right)\left[\begin{array}{cc}
s_{22} & -k_{21} \\
-k_{21} & k_{11}
\end{array}\right] \text {. }
$$

Em particular, esta última expressão será útil na análise de convergência e estabilidade do sistema em malha fechada. Finalmente, substituindo (35) e (39) em (34) e sabendo que $\tilde{v}=v-v^{*}$, a lei de controle ideal é dada por:

$$
\begin{aligned}
v_{1}^{*}= & t_{12} v_{2}+\lambda \operatorname{det}\left(K_{p}^{-1}\right) s_{22}\left(r_{c 1}-x_{c 1}\right) \\
& -\lambda \operatorname{det}\left(K_{p}^{-1}\right) k_{21}\left(r_{c 2}-x_{c 2}\right), \\
v_{2}^{*}= & -\lambda \operatorname{det}\left(K_{p}^{-1}\right) k_{21}\left(r_{c 1}-x_{c 1}\right) \\
& -\lambda \operatorname{det}\left(K_{p}^{-1}\right) k_{11}\left(r_{c 2}-x_{c 2}\right) .
\end{aligned}
$$

A partir das equações (40) e (41), obtém-se a seguinte parametrização linear:

$$
\begin{aligned}
& v_{1}=\theta_{13} v_{2}+\theta_{11}\left(r_{c 1}-x_{c 1}\right)+\theta_{12}\left(r_{c 2}-x_{c 2}\right), \\
& v_{2}=\theta_{21}\left(r_{c 1}-x_{c 1}\right)+\theta_{22}\left(r_{c 2}-x_{c 2}\right),
\end{aligned}
$$

donde é possível calcular os parâmetros ideais $\theta_{i j}^{*}$

$$
\begin{aligned}
& \theta_{13}^{*}=k_{11}^{-1}\left(k_{21}-k_{12}\right), \quad \theta_{21}^{*}=-\lambda \operatorname{det}\left(K_{p}^{-1}\right) k_{21}, \\
& \theta_{11}^{*}=\lambda \operatorname{det}\left(K_{p}^{-1}\right) s_{22}, \quad \theta_{22}^{*}=\lambda \operatorname{det}\left(K_{p}^{-1}\right) k_{11}, \\
& \theta_{12}^{*}=-\lambda \operatorname{det}\left(K_{p}^{-1}\right) k_{21} .
\end{aligned}
$$

De (42) é possível definir os vetores de parâmetros por $\theta_{1}=$ $\left[\begin{array}{lll}\theta_{13} & \theta_{11} & \theta_{12}\end{array}\right]^{T}$ e $\theta_{2}=\left[\begin{array}{ll}\theta_{21} & \theta_{22}\end{array}\right]^{T}$ e os vetores regressores por

$$
w_{1}=\left[\begin{array}{c}
\theta_{2}^{T} w_{2} \\
r_{c 1}-x_{c 1} \\
r_{c 2}-x_{c 2}
\end{array}\right], \quad w_{2}=\left[\begin{array}{c}
r_{c 1}-x_{c 1} \\
r_{c 2}-x_{c 2}
\end{array}\right],
$$

ou ainda, por

$$
\begin{aligned}
w_{1} & =w_{m 1}-\left[\begin{array}{c}
\theta_{2}^{T} e_{c}+\tilde{\theta}_{2}^{T} w_{m 2} \\
e_{c}
\end{array}\right], \\
w_{m 1} & =\left[\begin{array}{c}
\theta_{2}^{* T} w_{m 2} \\
r_{c}-x_{c d}
\end{array}\right], \\
w_{2} & =w_{m 2}-e_{c}, \quad w_{m 2}=r_{c}-x_{c d},
\end{aligned}
$$

sendo $w_{m 1}$ e $w_{m 2}$ sinais uniformemente limitados. Nota-se aqui a necessidade de utilizar uma quantidade maior de parâmetros do que a usualmente requerida em (9), dando origem a terminologia sobre-parametrização.

Com base em (42) e (43), a equação de erro (34) pode ser escrita na forma:

$$
\begin{aligned}
& \dot{e}_{c 1}=-\lambda e_{c 1}+k_{11} \tilde{\theta}_{1}^{T} w_{1}+k_{21} \tilde{\theta}_{2}^{T} w_{2}, \\
& \dot{e}_{c 2}=-\lambda e_{c 2}+k_{21} \tilde{\theta}_{1}^{T} w_{1}+k_{22} \tilde{\theta}_{2}^{T} w_{2},
\end{aligned}
$$

onde $\tilde{\theta}_{1}=\theta_{1}-\theta_{1}^{*}$ e $\tilde{\theta}_{2}=\theta_{2}-\theta_{2}^{*}$, ou, de maneira mais compacta

$$
\dot{e_{c}}=-\lambda e_{c}+S \tilde{v} .
$$

Analisando a estrutura da equação de erros acima, as leis de adaptação para $\tilde{\theta}_{1}$ e $\tilde{\theta}_{2}$ são dadas por:

$$
\begin{aligned}
& \dot{\theta}_{1}=\dot{\tilde{\theta}}_{1}=-\operatorname{sign}\left(s_{11}\right) \gamma w_{1} e_{c 1}, \\
& \dot{\theta}_{2}=\dot{\tilde{\theta}}_{2}=-\operatorname{sign}\left(s_{22}\right) \gamma w_{2} e_{c 2} .
\end{aligned}
$$

Considere o sistema adaptativo descrito por (5) e (6) com lei de controle dada por (42) e lei de adaptação dada por (50) e (51). Assume-se que o sinal de referência $r_{c}(t)$ é contínuo por partes e uniformemente limitado em norma. Então, o seguinte teorema pode ser enunciado.

Teorema 2 Se o ângulo de rotação $\phi \in(-\pi / 2, \pi / 2), k_{11}>$ 0 e $\operatorname{det}\left(K_{p}\right)>0$, então todos os sinais do sistema são uniformemente limitados e $e_{c}(t) \in \mathcal{L}_{2} \cap \mathcal{L}_{\infty} \lim _{t \rightarrow \infty} e_{c}(t)=0$.

Prova: Considere a função de Lyapunov candidata

$$
2 V=e_{c}^{T} S^{-1} e_{c}+\gamma^{-1}\left(\tilde{\theta}_{1}^{T} \tilde{\theta}_{1}+\tilde{\theta}_{2}^{T} \tilde{\theta}_{2}\right) .
$$

Derivando (52) em relação ao tempo, tem-se que:

$$
\dot{V}=e_{c}^{T} S^{-1} \dot{e_{c}}+\gamma^{-1}\left(\tilde{\theta}_{1}^{T} \dot{\tilde{\theta_{1}}}+\tilde{\theta}_{2}^{T} \dot{\tilde{\theta_{2}}}\right) .
$$


Avaliando $\dot{V}$ ao longo das trajetórias de (47) e (48), e utilizando as leis de adaptação (50) e (51), vem

$$
\dot{V}=-\lambda e_{c}^{T} S^{-1} e_{c} \leq 0 .
$$

A partir (52) e (54) conclui-se que os vetores $\tilde{\theta}_{i}$ e $e_{c}$ são uniformemente limitados (u.1.). Da equação $e_{c}=x_{c}-x_{c d}$ verifica-se que $x_{c}$ é u.l.. Sabendo que $\tilde{\theta}_{i}=\theta_{i}-\theta_{i}^{*}(i=1,2)$, e que $\theta_{i}^{*}$ são constantes, conclui-se que $\theta_{i}$ também são u.l.. De (43) tem-se que $\omega_{1}$ e $\omega_{2}$ são u.l. e consequentemente os sinais de controle dados por (42). Deste modo, das equações (47) e (48), conclui-se que $\dot{e_{c}}$ também é u.1.. Como $V$ é uniformemente limitada e $\dot{V}$ é uniformemente contínua $\left(\ddot{V}=-2 \lambda e_{c}^{T} S^{-1} \dot{e}_{c}\right.$ é limitada), então pelo Lema de Barbalat tem-se que $\lim _{t \rightarrow \infty} \dot{V}(t)=0$ e consequentemente que $\lim _{t \rightarrow \infty} e_{c}(t)=0$.

\section{ROBUSTEZ À INCERTEZAS CINEMÁTI- CAS}

No método apresentado em (Hsu e Aquino, 1999) a posição cartesiana $x$ poderia ser calculada através da transformação cinemática direta, já que os ângulos das juntas são mensuráveis e a cinemática do manipulador é assumida conhecida. Então a relação $x=K_{p}^{-1} x_{c}$ poderia ser usada e $v^{*}$ seria expressa em termos de $x$ ao invés de $x_{c}$, isto é, $v^{*}=\lambda K_{p}^{-1} r_{c}-\lambda x$. Entretanto, isso conduz a uma imperfeição na definição do controlador dada a presença de incertezas na cinemática do manipulador.

De fato, mesmo conhecendo perfeitamente $K_{p}$ (câmera calibrada) e sem adaptação, o erro na cinemática pode levar a servovisão imperfeita, mesmo para tarefas de regulação simples $\left(x_{c d} \equiv 0\right)$. Neste caso, o erro dinâmico pode ser expresso como:

$$
\dot{e}_{c}=K_{p} J(q)\left(\hat{J}(q) K_{p}\right)^{-1}\left[-\lambda\left(e_{c}-K_{p} \Delta F\right)\right],
$$

onde $\hat{J}$ representa o Jacobiano nominal e $\Delta F$ é a incerteza cinemática progressiva, isto é, $x=F(q)+\Delta F$. Expressando $K_{p} J(q)\left(\hat{J}(q) K_{p}\right)^{-1}=I+\Delta A$, tem-se que:

$$
\dot{e}_{c}=-\lambda(I+\Delta A) e_{c}+\lambda K_{p}(I+\Delta A) \Delta F,
$$

onde o termo $\Delta F$ não desaparece e seu efeito não é atenuado por $\lambda$. A razão principal é que, neste algoritmo, nenhuma realimentação direta do erro da imagem foi utilizada. Nos esquemas das Seções 3.1 e 4, tem-se que a equação do erro é dada por:

$$
\dot{e}_{c}=-\lambda K_{p} J(q)\left(\hat{J}(q) K_{p}\right)^{-1} e_{c}=-\lambda(I+\Delta A) e_{c},
$$

e então, para um pequeno $\Delta A$, o erro da imagem $e_{c}$ tende exponencialmente para zero.

\section{SIMULAÇÕES}

Para ilustrar as propriedades de robustez dos controladores adaptativos, são apresentados alguns resultados de simulação obtidos com um robô de dois elos, similar ao manipulador utilizado na implementação experimental. Os comprimentos dos elos são $l_{1}=279[\mathrm{~mm}] \mathrm{e} l_{2}=228[\mathrm{~mm}]$, para os elos $1 \mathrm{e}$ 2 , respectivamente. Os parâmetros do robô/controlador são: $\phi=\frac{\pi}{4}[\mathrm{rad}] ; h_{1}=\frac{f \alpha_{1}}{f-Z}=0.7$ [pixels $\left./ \mathrm{mm}\right] ; h_{2}=\frac{f \alpha_{2}}{f-Z}=$ 0.6 [pixels $/ \mathrm{mm}$ ]; $\lambda=1 ; \gamma=0.002$; Considerando uma incerteza de $10 \%$ em $l_{2}$ e assumindo que $\phi, h_{1}, h_{2}$ são conhecidos, a Figura 1 descreve o comportamento dos erros de rastreamento para os esquemas adaptativo hierárquico, adaptativo simétrico e hierárquico não-adaptativo, submetidos à mesma condição de incertezas cinemáticas.
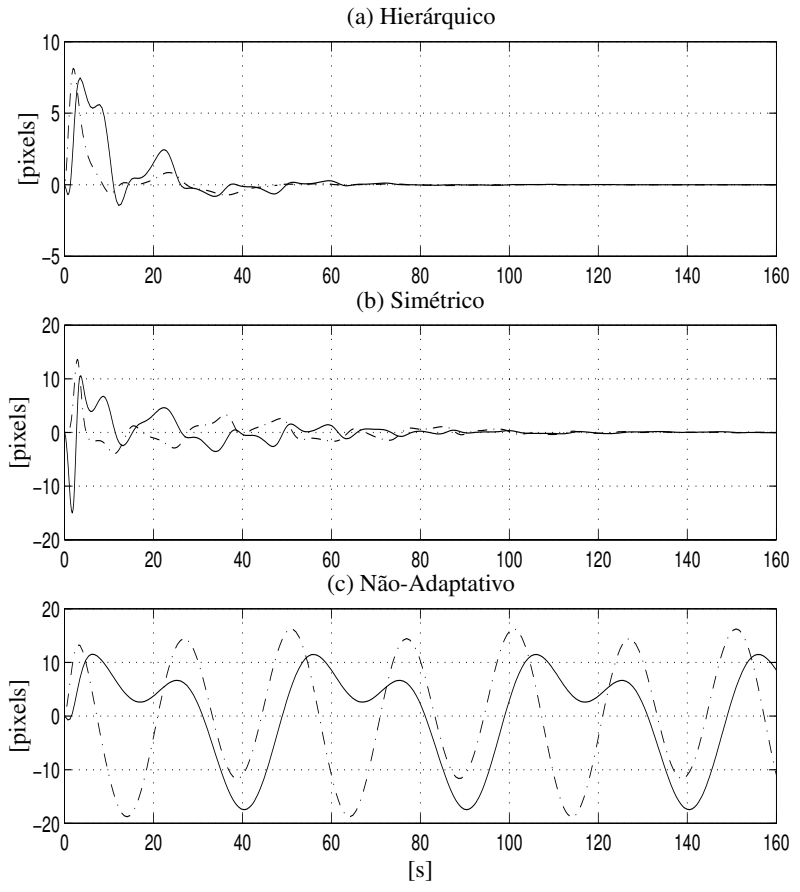

Figura 1: Resultados de Simulação - Erro de Rastreamento $e_{c}$. (-) $e_{c 1},(-.) e_{c 2}$.

\section{RESULTADOS EXPERIMENTAIS}

Nesta seção, são apresentados os resultados experimentais obtidos com a implementação dos controladores adaptativo hierárquico e adaptativo simétrico, propostos nas seções 3.1 e 4 respectivamente. 


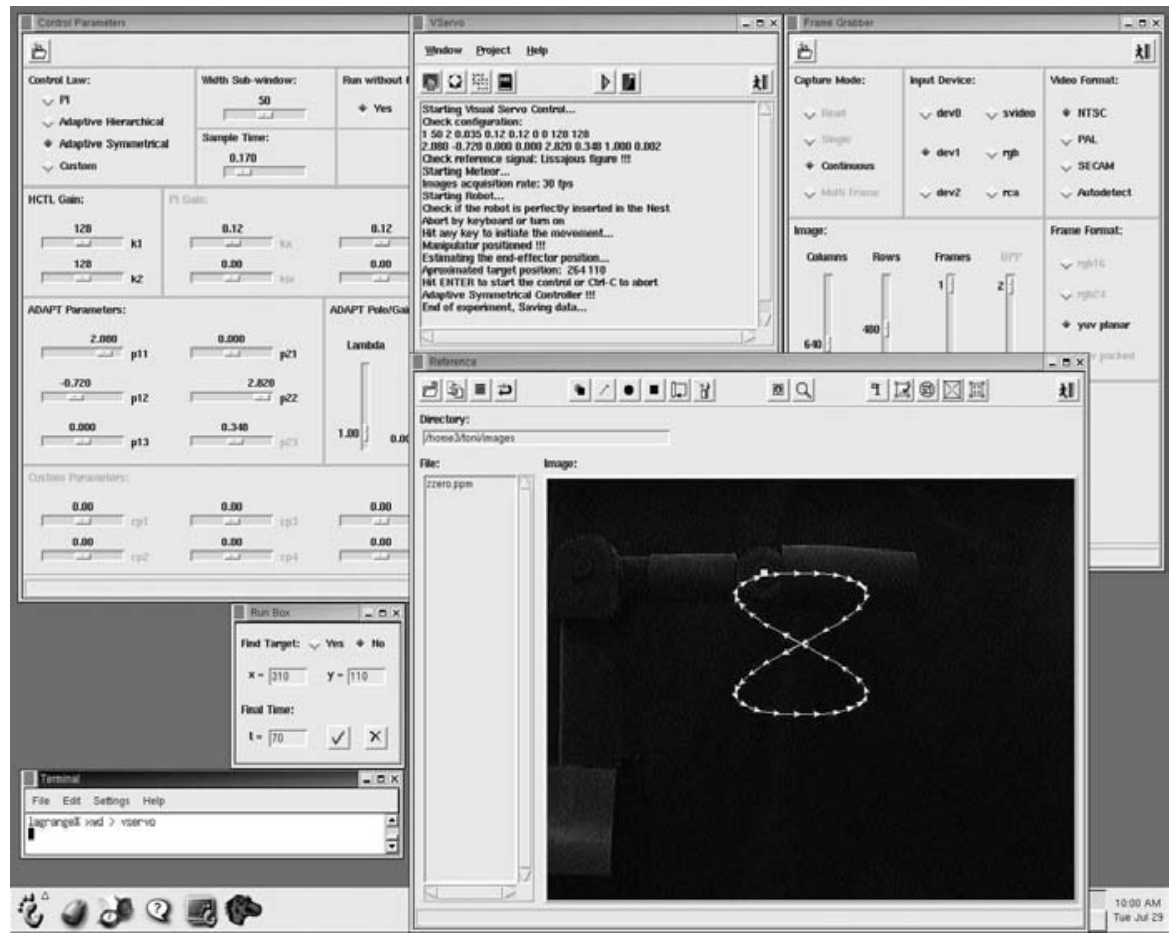

Figura 2: Interface Gráfica VServo.

\subsection{Ambiente de Trabalho}

As leis de controle foram implementadas em um manipulador robótico Zebra Zero (IMI Inc.) com 6 graus de liberdade movimentado-se em um plano vertical. Desta forma, são permitidos apenas movimentos das juntas 2 e 3 (ombro e cotovelo).

O sistema servovisual utiliza configuração de câmera fixa no espaço de trabalho: uma câmera CCD KP-D50 (Hitachi Ltd.) com distância focal $f=6[\mathrm{~mm}]$ foi montada em frente ao manipulador (vide Figura 2 para o ponto de vista da câmera). A característica visual extraída é a coordenada da imagem do centróide de um disco branco fixado no punho do robô. As imagens de tamanho $640 \times 480$ pixels são capturadas usando uma placa Frame-Grabber Meteor (Matrox Ltd.), com taxa de aquisição de 30 quadros por segundo (FPS) com 256 níveis de cinza. A primeira estimativa das coordenadas do disco branco é realizada off-line usando subtração de imagens. Para garantir que o disco permaneça sempre visível, o processamento de imagem é realizado em uma subjanela de dimensões $50 \times 50$ pixels.

Os comandos de velocidade das juntas, gerados a partir das leis de controle adaptativas, alimentam a placa de controle (ISA card) do Zebra Zero que fecha a malha de velocidade utilizando um microcontrolador HCTL1100 (HP Inc.), ope- rando em modo de velocidade proporcional com período de amostragem de $0.52 \mathrm{~ms}$.

Para evitar o erro de regime estacionário devido à gravidade, uma compensação de gravidade foi implementada usando um simples modelo (Spong e Vidyasagar, 1989) $(g(q)=$ $\left.\left[p_{1} \cos \left(q_{2}\right)+p_{2} \sin \left(q_{2}+q_{3}\right), p_{2} \sin \left(q_{2}+q_{3}\right)\right]\right)$ sintonizado experimentalmente.

A profundidade média entre o plano da imagem e o espaço de trabalho do robô foi $Z=1[\mathrm{~m}]$. Todos os testes foram planejados para evitar as singularidades no Jacobiano.

\subsection{Interface Gráfica}

Os controladores adaptativos propostos foram codificados em linguagem $\mathrm{C}$ e executados em um computador Pentium Pro 200 com Linux OS, através de uma Interface Gráfica com o Usuário desenvolvida em Tcl/Tk (Ousterhout, 1993).

A Interface Gráfica, denominada VServo (vide Figura 2), permite ao usuário configurar os parâmetros do sistema servovisual (controladores e frame-grabber), definir trajetórias de referência sob o ponto de vista da câmera e visualizar a trajetória seguida pelo manipulador após a realização da experiência. Além disso, através de VServo é possível executar rotinas em linguagem $C$ para aquisição de imagens, iniciali- 

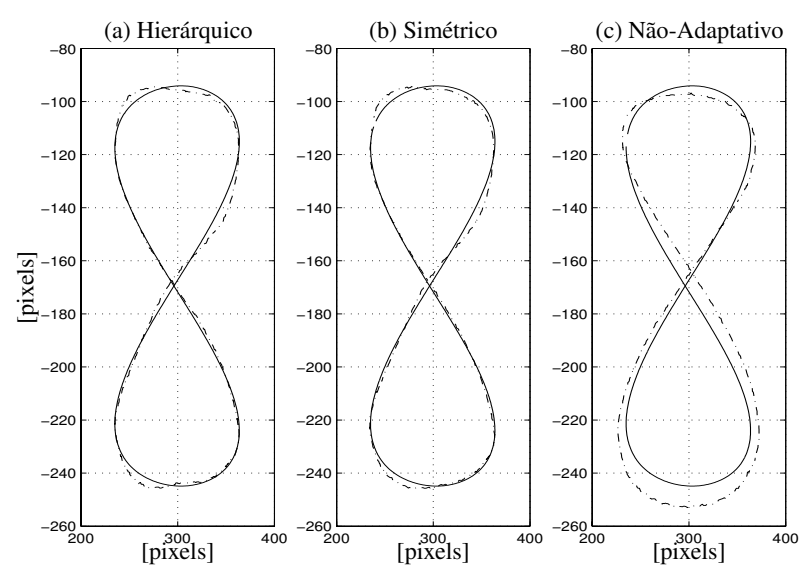

Figura 3: Resultados Experimentais - Rastreamento em regime. (-) Trajetória desejada $x_{c d},(-$.$) Trajetória atual x_{c}$.

zação do robô, localização do alvo fixado no punho do robô e controle servo visual do manipulador robótico.

\subsection{Análise dos Resultados}

Os resultados experimentais ilustrados nas Figuras 3-8, foram obtidos sem qualquer calibração prévia da câmera. A orientação da câmera é $\phi \approx 0$. As condições iniciais para os parâmetros adaptativo $\theta_{11}(0)=2.080[\mathrm{~mm} / \mathrm{s}$. pixels] $\theta_{12}(0)=-0.720[\mathrm{~mm} / \mathrm{s}$.pixels $], \theta_{13}(0)=0.000, \theta_{21}(0)=$ $0.000[\mathrm{~mm} / \mathrm{s}$. pixels $], \theta_{22}(0)=2.820[\mathrm{~mm} / \mathrm{s}$.pixels $] \mathrm{e}$ $\theta_{23}(0)=0.348$, foram obtidas a partir da melhor sintonia para o caso não-adaptativo (i.e. $\gamma=0$ ) $\operatorname{com} \phi \approx \frac{\pi}{4}$. Os parâmetros de controle utilizados foram: $\gamma=0.002, \lambda=1$ e sem projeção nas leis de adaptação (22) e (23).

As trajetórias $x_{c d}$ desejada (linha cheia) e $x_{c}$ do centróide (linha pontilhada) são mostradas na Figura 3. As Figuras 3(a) e 3(b) ilustram o rastreamento em regime para os controladores adaptativos hierárquico e simétrico respectivamente. $\mathrm{Na}$ Figura 3(c) pode-se observar a degradação do desempenho do sistema quando nenhum mecanismo de adaptação é utilizado no algoritmo de controle hierárquico (i.e. $\gamma=0$ ), no caso em que a câmera não está calibrada.

As Figuras 4, 5 e 6 mostram o erro de rastreamento $e_{c}$ e o sinal de controle cartesiano $v$, respectivamente. Pode-se observar que com os controladores adaptativos propostos $e_{c}$ tende para um pequeno conjunto residual da ordem de 1 pixel.

O comportamento dos parâmetros $\theta$ para o controlador adaptativo hierárquico é ilustrado na Figura 7 , onde é possível observar que os valores tenderam para $\theta_{11}=1.02$ $\left[\mathrm{mm} / \mathrm{s}\right.$.pixels], $\theta_{12}=0.00\left[\mathrm{~mm} / \mathrm{s}\right.$.pixels], $\theta_{22}=1.20$ $\left[\mathrm{mm} / \mathrm{s}\right.$.pixels] e $\theta_{23}=0.00$, após um período de adaptação.
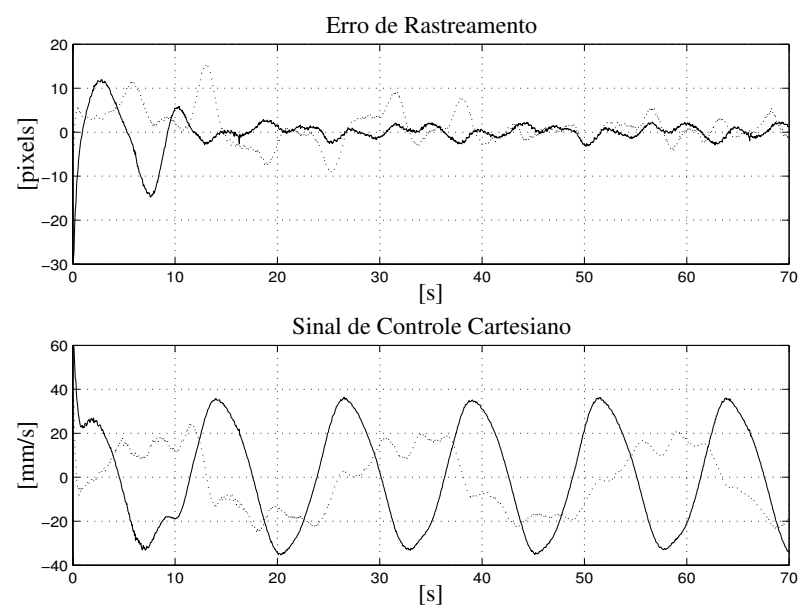

Figura 4: Resultado Experimental (Controlador Adaptativo Hierárquico) - Erro de Rastreamento $e_{c}$ e Sinal de Controle Cartesiano $v .(-) e_{c 1}$, (..) $e_{c 2},(-) v_{1}$, (..) $v_{2}$.
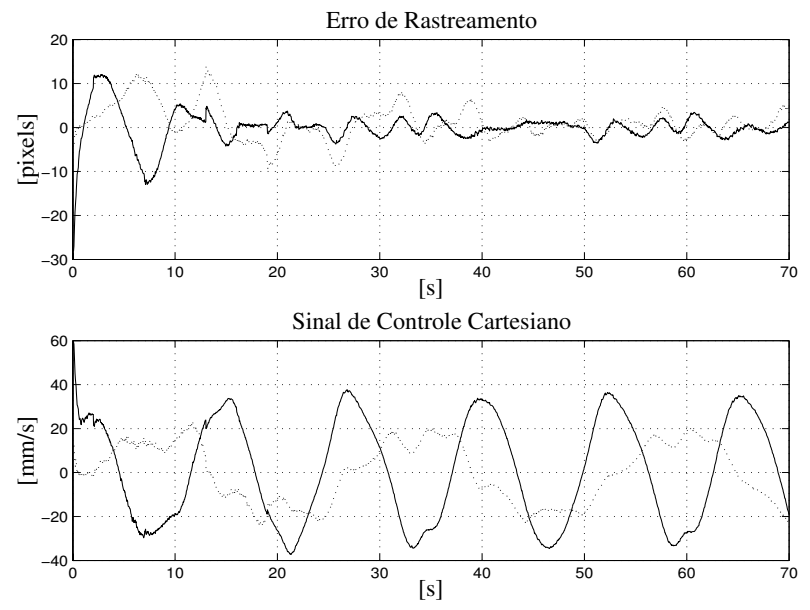

Figura 5: Resultado Experimental (Controlador Adaptativo Simétrico) - Erro de Rastreamento $e_{c}$ e Sinal de Controle Cartesiano $v .(-) e_{c 1}$, (..) $e_{c 2},(-) v_{1}$, (..) $v_{2}$.

A evolução do vetor de parâmetros $\theta$ para o controlador adaptativo simétrico é ilustrada na Figura 8 , onde pode-se observar que os valores em regime tenderam para $\theta_{11}=1.04$ $\left[\mathrm{mm} / \mathrm{s}\right.$.pixels], $\theta_{12}=-0.296[\mathrm{~mm} / \mathrm{s}$.pixels $], \theta_{13}=0.288$, $\theta_{21}=-0.136[\mathrm{~mm} / \mathrm{s}$.pixels $]$ e $\theta_{22}=1.18[\mathrm{~mm} / \mathrm{s}$.pixels $]$.

Comparando a Figura 3 com a posição do punho obtida através da cinemática direta a partir das medidas dos encoders do manipulador robótico, é possível observar a grande incerteza cinemática do robô utilizado devido à flexibilidade do braço e folga nas engrenagens das juntas.

Contudo, a hipótese que o eixo ótico é perpendicular ao es- 

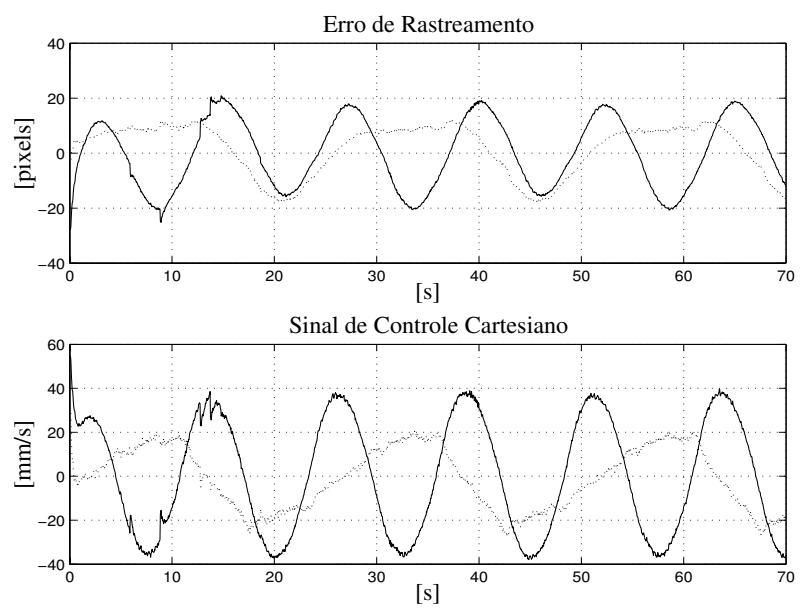

Figura 6: Resultado Experimental (Controlador NãoAdaptativo) - Erro de Rastreamento $e_{c}$ e Sinal de Controle Cartesiano $v$. (-) $e_{c 1}$, (..) $e_{c 2},(-) v_{1}$, (..) $v_{2}$.

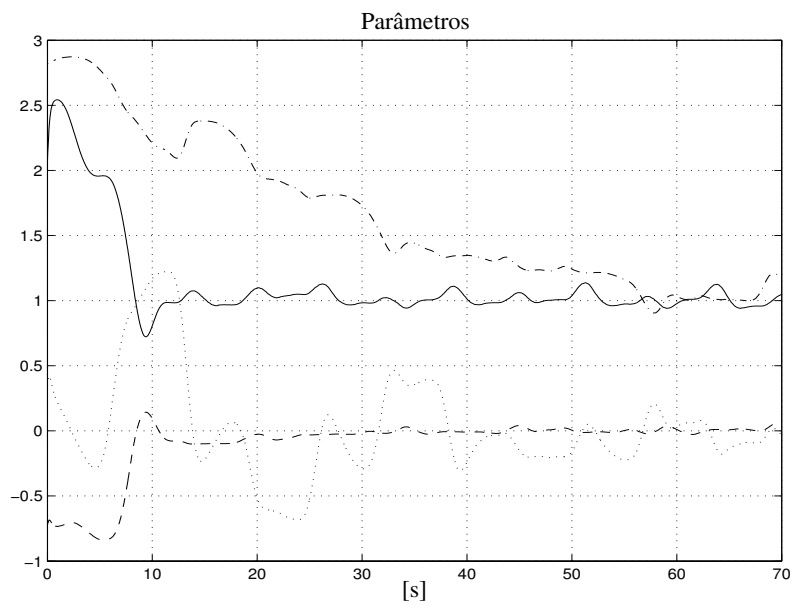

Figura 7: Resultado Experimental (Controlador Adaptativo Hierárquico) - Parâmetros. (-) $\theta_{11}$, (- -) $\theta_{12}$, (-.-) $\theta_{22}$, (..) $\theta_{23}$.

paço de trabalho do robô não é perfeitamente satisfeita. Apesar das incertezas cinemáticas, os controladores adaptativos propostos garantem excelente desempenho e robustez.

\subsection{Comparação entre os métodos}

Com relação ao sistema de servovisão abordado, observa-se que o controlador adaptativo, projetado pelo método de $\mathrm{Si}$ metrizacão de $K_{p}(\mathrm{SK})$, apresenta desempenho semelhante ao do controlador projetado pelo método de Hierarquia de Controle (HC). As diferenças entre os dois esquemas propostos podem ser verificadas sob dois aspectos: análise de

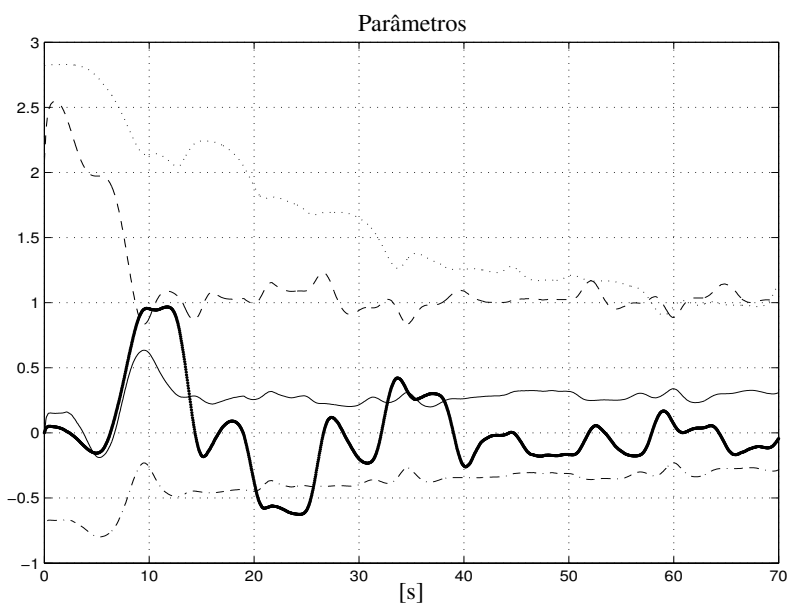

Figura 8: Resultado Experimental (Controlador Adaptativo Simétrico) - Parâmetros. (- -) $\theta_{11},(-.-) \theta_{12},(-) \theta_{13}$, (.) $\theta_{21}$, (..) $\theta_{22}$

estabilidade e complexidade ${ }^{4}$ do controlador. A análise de convergência e estabilidade, para o sistema projetado via hierarquia de controle, é mais complexa e envolve análise funcional (Hsu e Costa, 1999); para o sistema projetado via simetrização é possível definir uma simples função de Lyapunov. Contudo, a estrutura obtida para o controlador adaptativo hierárquico é mais simples pois não envolve sobreparametrização.

Um tópico de pesquisa atual é a comparação dos métodos propostos neste trabalho com um método recentemente proposto em (Astolfi, Hsu, Netto e Ortega , 2002) usando a abordagem I\&I (Imersion and Invariance) e um termo PI nãolinear, que em princípio não requer sobre-parametrização da lei de controle, projeção na lei de adaptação ou condição de excitação persistente.

\section{CONCLUSÕES}

Foram propostas duas estratégias adaptativas para rastreamento visual em sistemas robóticos com incertezas nos parâmetros da câmera. A análise de estabilidade e convergência para os sistemas em malha fechada foi apresentada. Resultados experimentais em um manipulador robótico planar ilustram o desempenho equivalente dos esquemas propostos.

Os tópicos de pesquisa no futuro de acordo com as idéias desenvolvidas aqui são: aplicação em navegação autônoma de veículos por servovisão, extensão para os problemas de servovisão stereo 3D com câmera fixa ou em movimento (e.g., a configuração eye-in-hand), generalizações para con-

\footnotetext{
${ }^{4}$ Aqui, a terminologia complexidade refere-se a quantidade de termos que compõem o controlador.
} 
trole dinâmico do robô e aplicações em controle híbrido de força/visão.

\section{AGRADECIMENTOS}

Este trabalho foi parcialmente financiado pelo CNPq.

\section{REFERÊNCIAS}

Astolfi A., Hsu, L., Netto, M.S, e Ortega, R., (2002). Two solutions to the Adaptive Visual Servoing Problem, IEEE Trans. Robotics and Automation, vol. 18, no. 3, pp. 387-392.

Bishop, B. e Spong, M. (1997). Adaptive calibration and control of 2D monocular visual servo systems, Proc. of SYROCO-97, (Nantes).

Hosoda, K., Igarashi, K. e Asada, M. (1998). Adaptive Hybrid Control for Visual and Force Servoing in an Unknown Environment, IEEE Robotics and Automation Magazine, (December), pp. 39-43.

Hsu, L. e Aquino, P. (1999). Adaptive visual tracking with uncertain manipulator dynamics and uncallibrated camera, Proc. IEEE Conf. on Dec. and Contr., (Phoenix), pp. 1248-1253.

Hsu, L. e Costa, R. (1999). MIMO direct adaptive control with reduced prior knowledge of the high frequency gain, Proc. IEEE Conf. on Dec. and Contr., (Phoenix), pp. 3303-3308.

Hsu, L., Costa, R. e Imai, A. (1999). Stable adaptive control of MIMO systems via symmetrization of the high frequency gain matrix, Technical Report, PEE/COPPE, UFRJ.

Hsu, L. e Lizarralde, F. (2000). Robust Adaptive Visual Tracking Control: analysis and experiments, em Proc. Conf. on Control and Applications, (Anchorage).

Hsu, L., Zachi, A. R. L. e Lizarralde, F. (2001). Adaptive Visual Tracking for Motions on Smooth Surfaces, Proc. IEEE Conf. on Dec. and Contr., (Orlando), pp. 24302435.

Hsu, L., Leite, A. C. e Lizarralde, F. (2002). Controle Adaptativo Robusto para Rastreamento Visual, XIV Congresso Brasileiro de Automática, (Natal), Brasil, pp. 661-666.

Hutchinson, S., Hager, G. e Corke, P. (1996). A tutorial on visual servo control, IEEE Trans. Robotics and Automation, vol. 12 , no. 5, pp. 651-670.
Ioannou, P. e Sun, K. (1996). Robust Adaptive Control. Prentice Hall, 1996

Kelly, R., (1996). Robust asymptotically stable visual servoing of planar robots, IEEE Trans. Robotics and Automation, vol. 12, no. 5, pp. 759-766.

Kelly, R., Reyes, R., Moreno, J., e Hutchinson, S. (1999). A two loops direct visual control of direct-drive planar robots with moving target, Proc. IEEE Conf. Robotics \& Automation, (Detroit), pp. 599-604.

Khalil, H., (1996). Nonlinear Systems. Prentice Hall, 2 ed., 1996

Koivo, A e Houshangi, N.(1991). Real-time vision feedback for servoing of a robotic manipulator with self-tuning controller, IEEE Trans. Syst. Man, Cybern., vol. 21, pp. 134-142.

Michel, R. e Miller, A. (1982). Ordinary Differential Equation. Academic Press, 1982.

Narendra, K. e Annaswamy, A. (1989). Stable Adaptive Systems. Prentice Hall, 1989

Ousterhout, J. K., (1993). Tcl and the Tk Toolkit. AddisonWesley, 1 ed., 1993.

Papanikolopoulos, N. e Khosla, P. (1993). Adaptive robotic visual tracking: Theory and experiments, IEEE Trans. Aut. Contr., vol. 38, no. 3, pp. 429-445.

Sastry, S. S e Bodson, M. (1989). Adaptive Control: Stability, Convergence and Robustness. Prentice Hall, 1989.

Slotine, J. J. E. e Li, W. (1991). Applied Nonlinear Control. Prentice Hall, 1991.

Spong, M. V e Vidyasagar, M.(1989). Robot Dynamics and Control. John Wiley\&Sons.

Zergeroglu, E., Dawson, D., de Queiroz, M. e Nagarkatti, S. (1999). Robust visual-servo control of robot manipulators in the presence of uncertainty, Proc. IEEE Conf. Dec. and Contr., (Phoenix), pp. 4137-4142. 